Reprod. Nutr. Dévelop., 1983, 23 (6), 995-1002.

\title{
Synthèse d'œstrogènes par l'ovaire embryonnaire de Poulet à partir de divers précurseurs radioactifs : comparaison des taux de synthèse
}

\author{
J.-P. WENIGER, J. CHOURAQUI, A. ZEIS
}

Laboratoire de Zoologie et d'Embryologie expérimentale, Université Louis-Pasteur.

12, rue de l'Université, 67000 Strasbourg.

Summary. A study of oestrogen synthesis by the chick embryo ovary using various radioactive precursors.

The ability of the 17-day old chick embryo ovary to convert radioactive progesterone, testosterone, androstenedione and dehydroepiandrosterone to oestrone and oestradiol was investigated. The conversion rate was highest with dehydroepiandrosterone $(13 \%)$. Testosterone was the next best precursor $(6.5 \%)$, closely followed by androstenedione $(5.1 \%)$, with progesterone giving the lowest rate $(0.9 \%)$. The synthesis of oestrogens from dehydroepiandrosterone thus seemed to bypass testosterone and androstenedione.

The level of oestradiol synthesis was consistently higher than that of oestrone. Measurable testosterone was not formed from dehydroepiandrosterone. All these results have been discussed in relation with previously published data.

\section{Introduction.}

La capacité de synthèse d'œstrone et d'œstradiol par l'ovaire embryonnaire de Poulet à partir de divers précurseurs exogènes, radioactifs ou non, a déjà fait l'objet de plusieurs travaux (Haffen et Cédard, 1968 ; Weniger, 1969 ; Guichard et al., 1973 et 1979b). Mais il manquait une étude systématique visant la comparaison des taux de synthèse. Une telle étude n'était pas superflue, puisque les données éparses dans la littérature étaient obtenues avec des précurseurs marqués les uns au ${ }^{3} \mathrm{H}$, les autres au ${ }^{14} \mathrm{C}$, d'activité spécifique très différente, ou se rapportaient à des stades embryonnaires différents. De plus, les résultats présentaient certaines discordances, suivant que les précurseurs étaient utilisés à l'état radioactif ou non. Par exemple, la synthèse d'œstradiol était nettement plus 
importante que celle d'œstrone avec la déhydroépiandrostérone- ${ }^{14} \mathrm{C}$ (Haffen et Cédard, 1967), alors que c'était l'inverse avec le même précurseur à l'état non radioactif (Guichard et al., 1979b). Nous avons donc étudié la synthèse d'œstrone et d'œstradiol par l'ovaire embryonnaire de Poulet à un même stade de 17 jours à partir de précurseurs tritiés d'une même activité spécifique. Nous nous sommes aussi intéressés à la synthèse de testostérone.

\section{Matériel et méthodes.}

Matériel biologique. - Les embryons de Poulet utilisés étaient de race Leghorn blanche et avaient 17 jours. L'importance de la production de stéroïdes par les gonades embryonnaires semblant dépendre de la saison (Guichard et al., 1979a), indiquons que les œufs utilisés pour la 1re expérience avaient été pondus en mai, ceux utilisés pour la $5^{\mathrm{e}}$ expérience en octobre et ceux utilisés pour les $2^{\mathrm{e}}$, $3^{\mathrm{e}}$ et $4^{\mathrm{e}}$ expériences en mars.

Culture d'organes. - Les ovaires gauches furent découpés en cinq ou six avant d'être cultivés in vitro pendant 24 h sur le milieu gélosé de Wolff et Haffen (1952) en présence du précurseur radioactif choisi.

Précurseurs radioactifs. - Les précurseurs radioactifs utilisés étaient la progestérone - $1,2,6,7-3 \mathrm{H}$, l'androstènedione $-1,2,6,7-3 \mathrm{H}$ et la déhydroépiandrostérone - 1, 2, 6, 7-3 $\mathrm{H}$, qui provenaient du Radiochemical Centre (Amersham), et la testostérone - 1, 2, 6, 7-3 $\mathrm{H}$, qui provenait du CEA (Gif-surYvette). Leur activité spécifique était respectivement de $85,74,67$ et $83 \mathrm{Ci} / \mathrm{mmol}$. Leur pureté radiochimique, vérifiée par chromatographie sur couche mince, était égale ou supérieure à $95 \%$.

Ils furent préparés en solution dans le mélange propanediol-liquide de Tyrode $1: 3$ à la concentration radioactive de $0,30-0,37 \mu \mathrm{Ci} / \mu \mathrm{l}$, correspondant à une concentration massique voisine de $1,5 \mathrm{ng} / \mu \mathrm{l}$ ou à une concentration molaire approximativement $5 \mu \mathrm{M}$. Les ovaires étant explantés sur le milieu de culture, chacun d'eux fut baigné dans une gouttelette $d^{\prime} 1 \mu$ de la solution radioactive. Les conditions particulières à chaque expérience sont précisées dans le tableau 2.

Traceurs radioactifs. - Afin de déterminer les pertes survenant au cours de l'analyse, les milieux de culture ont été additionnés de quantités traceuses d'œstrone-4- ${ }^{14} \mathrm{C}$ et d'œstradiol-4- ${ }^{14} \mathrm{C}$ de l'ordre de 20000 dés/min au début de l'analyse. Dans une expérience, la testostérone-4- ${ }^{14} \mathrm{C}$ a été ajoutée en plus. Les 3 traceurs provenaient de New England Nuclear (Boston) et étaient radiochimiquement purs à $97 \%$.

Extraction des cstrogènes et méthylation. - Les œestrogènes n'étant pratiquement pas extractibles des milieux de culture par l'éther éthylique après simple homogénéisation mécanique (Akram et Weniger, 1973), on les soumet à une hydrolyse acide $(15 \% \mathrm{HCl})$ à l'ébullition avec reflux pendant $1 \mathrm{~h}$. Au préalable, on a ajouté aux milieux $100 \mu \mathrm{g}$ d'œstrone et $100 \mu \mathrm{g}$ d'œstradiol non radioactifs comme entraîneurs, ainsi que les traceurs marqués au ${ }^{14} \mathrm{C}$. L'hydrolysat est extrait par l'éther éthylique, l'extrait éthéré est lavé et évaporé à sec. Le résidu de l'éva- 
poration est repris par le mélange benzène-éther de pétrole $1: 1$, d'où les œstrogènes sont extraits par la soude $0,4 \mathrm{~N}$, les stéroïdes neutres restant dans la phase organique. Les œstrogènes sont purifiés par méthylation (Brown, 1955), puis chromatographiés.

Chromatographie. - Les éthers méthyliques de l'œstrone et de l'œstradiol sont séparés par chromatographie sur couche mince de gel de silice dans le système chloroforme-éther $9: 1$ et repérés aux rayons ultraviolets. Ils sont élués et rechromatographiés dans le système cyclohexane-acétate d'éthyle $7: 3$, l'œstrone méthylée après réduction en œstradiol méthylé. L'évaluation quantitative se fait après recristallisations à activité spécifique constante.

Recristallisations. - Le dernier éluat est évaporé à sec et additionné de $15 \mathrm{mg}$ d'œstradiol méthylé non radioactif, après quoi on procède à une série de recristallisations, utilisant alternativement les systèmes de solvants méthanoldichlorométhane-éther de pétrole et acétone-chloroforme-éther de pétrole. Après chaque recristallisation, on recueille les eaux-mères, qu'on sèche et pèse. On pèse aussi un échantillon de cristaux, de l'ordre d'1 mg. La série de recristallisations terminée, cristaux et eaux-mères sont repesés avec précision à $0,008 \mathrm{mg}$ près et dissous dans le mélange scintillant en vue de la mesure de la radioactivité.

Mesure de la radioactivité. - Elle se fait à l'aide d'un spectromètre à scintillation dont les fenêtres sont réglées de telle sorte à éviter tout passage du ${ }^{3} \mathrm{H}$ dans la voie du ${ }^{14} \mathrm{C} .15 \%$ de ${ }^{14} \mathrm{C}$ passent dans la voie du ${ }^{3} \mathrm{H}$ et sont déduits de l'activité dans cette voie. Dans ces conditions, le rendement du comptage est de $37 \%$ pour le ${ }^{3} \mathrm{H}$ et de $67 \%$ pour le ${ }^{14} \mathrm{C}$, le bruit de fond étant respectivement de 12 et 15 coups $/ \mathrm{min}$. Les échantillons sont comptés le temps qu'il faut pour réduire l'incertitude sur le taux de comptage au seuil de $5 \%$ à moins de $3 \%$.

Critère d'identification des cestrogènes et évaluation quantitative. - Dans ces conditions, l'activité spécifique sera déterminée avec une incertitude inférieure à $5 \%$. Conformément au critère établi par Axelrod et al. (1965), elle sera considérée comme constante si les valeurs obtenues au cours de 3 recristallisations successives s'écartent de moins de $5 \%$ de la valeur moyenne. La valeur constante de l'activité spécifique par rapport au ${ }^{14} \mathrm{C}$ permettra de calculer le pourcentage de récupération, celle constante par rapport au ${ }^{3} \mathrm{H}$ le pourcentage de transformation. Le tableau 3 illustre ces opérations.

Identification de la testostérone. - Isolée de la phase benzène-éther de pétrole par chromatographie, la testostérone fut acétylée et l'acétate de testostérone recristallisé avec $20 \mathrm{mg}$ d'entraîneur non radioactif dans les mêmes systèmes de solvants que l'œstradiol méthylé.

Expression des résultats. - Les résultats sont donnés en pourcentages de transformation lou taux de synthèse) d'une part, en quantités d'œstrogènes synthétisées de l'autre.

\section{Résultats.}

II importait de vérifier dans une expérience préliminaire si œstrogènes marqués au ${ }^{3} \mathrm{H}$ et œestrogènes marqués au ${ }^{14} \mathrm{C}$ subissaient les mêmes pertes au cours 
TABLEAU 1

Comparaison des pourcentages de récupération de l'cestradiol marqué par le ${ }^{3} \mathrm{H}$ et de l'cestradiol marqué par le ${ }^{14} \mathrm{C}$.

Activité spécifique (A.S.) exprimée en coups $/ \mathrm{min} / \mathrm{mg}$. Les valeurs constantes sont encadrées.

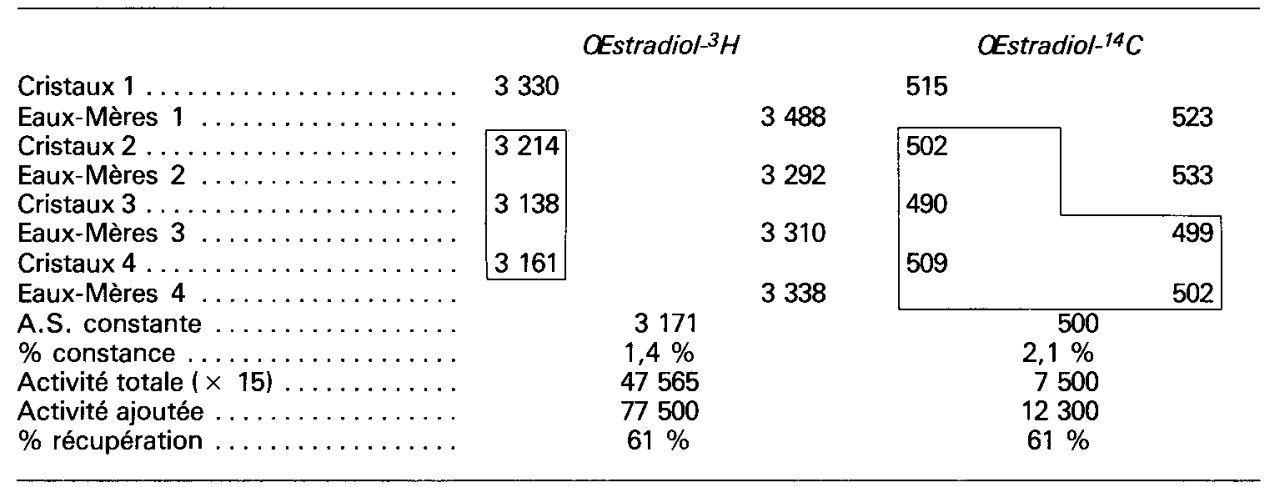

de l'analyse. Ce n'est en effet qu'à cette condition que l'addition d'une quantité traceuse d'œstrogène marqué au ${ }^{14} \mathrm{C}$ pourrait servir à la détermination du pourcentage de récupération de l'œstrogène synthétisé à partir du précurseur tritié. Nous avons effectué cette vérification sur l'œstradiol, ajoutant à des milieux de culture vierges des quantités connues d'œstradiol-6, $7-{ }^{3} \mathrm{H}$ et d'œstradiol-4- ${ }^{-14} \mathrm{C}$ avant l'hydrolyse et parcourant ensuite tout le mode opératoire jusqu'aux recristallisations incluses. Le tableau 1 montre que les 2 pourcentages de récupération sont identiques. La détermination du pourcentage de récupération de l'œtrogène tritié synthétisé par l'intermédiaire de celui de l'œstrogène marqué au ${ }^{14} \mathrm{C}$ est donc licite.

En dehors des pertes survenant au cours de l'analyse, il faudrait encore tenir compte dans le calcul du pourcentage de transformation du précurseur en œstrogène de la perte de ${ }^{3} \mathrm{H}$ dans les positions $1 \beta$ et $2 \beta$ qu'entraîne l'aromatisation. Mais la proportion de ${ }^{3} \mathrm{H}$ fixé en $1 \beta$ et $2 \beta$ ne dépassait pas $15 \%$ dans le cas le plus défavorable, celui de la déhydroépiandrostérone, de telle sorte que cette perte supplémentaire a été négligée.

Afin de pouvoir comparer valablement les taux de synthèse d'œstrone et d'œstradiol, il était nécessaire que les précurseurs eussent une même activité spécifique et qu'ils fussent utilisés en quantité équivalente à une même concentration. Ces conditions ont été remplies aussi bien que possible, comme le montre le tableau 2. L'examen de ce même tableau autorise en outre les conclusions suivantes : 1) Le taux de synthèse le plus élevé a été obtenu avec la déhydroépiandrostérone. Suivent dans l'ordre la testostérone et l'androstènedione, qui se tiennent d'assez près, la progestérone se détachant assez nettement. 2) La synthèse d'œstradiol a toujours été sensiblement plus importante que celle d'œstrone.

A titre d'exemple, le tableau 3 donne les résultats détaillés de l'expérience 5, où la testostérone avait été recherchée en plus de l'œstrone et de I'œstradiol. L'activité spécifique de la testostérone par rapport au ${ }^{3} \mathrm{H}$ tendant vers zéro, celleci n'est donc pas identifiée. 
TABLEAU 2

Conditions expérimentales et taux de synthèse d'œstrone $\left(E_{1}\right)$ et d'œstradiol $\left(E_{2}\right)$.

\begin{tabular}{|c|c|c|c|c|c|c|c|c|c|c|}
\hline \multirow{2}{*}{$\begin{array}{l}\text { Expé- } \\
\text { rience }\end{array}$} & \multirow{2}{*}{$\begin{array}{l}\text { Pré- } \\
\text { curseur }\end{array}$} & \multirow{2}{*}{$\begin{array}{c}\text { Activité } \\
\text { spécifique } \\
\text { (Ci/mmol) }\end{array}$} & \multirow{2}{*}{$\begin{array}{c}\text { Concentration } \\
\text { radioactive } \\
(\mu \mathrm{Ci} / \mu \mid)\end{array}$} & \multirow{2}{*}{$\begin{array}{c}\text { Nombre } \\
\text { d'ovaires }\end{array}$} & \multicolumn{3}{|c|}{$\begin{array}{c}\text { Taux de synthèse } \\
(\%)\end{array}$} & \multicolumn{3}{|c|}{$\begin{array}{l}\text { Quantités formées } \\
\text { (fmol/ovaire) }\end{array}$} \\
\hline & & & & & $E_{1}$ & $E_{2}$ & $E_{1}+E_{2}$ & $E_{1}$ & $E_{2}$ & $E_{1}+E_{2}$ \\
\hline $\begin{array}{l}1 \\
2 \\
3 \\
4 \\
5\end{array}$ & $\begin{array}{c}\text { Pro } \\
\text { T } \\
\text { A } \\
\text { DHA } \\
\text { DHA }\end{array}$ & $\begin{array}{l}85 \\
83 \\
74 \\
67 \\
66\end{array}$ & $\begin{array}{l}0,30 \\
0,37 \\
0,36 \\
0,31 \\
0,37\end{array}$ & $\begin{array}{r}13 \\
4 \\
5 \\
5 \\
3\end{array}$ & $\begin{array}{l}0,26 \\
1,3 \\
1,4 \\
4,4 \\
4,7\end{array}$ & $\begin{array}{l}0,61 \\
5,2 \\
3,7 \\
9,6 \\
7,3\end{array}$ & $\begin{array}{r}0,9 \\
6,5 \\
5,1 \\
14,0 \\
12,0\end{array}$ & $\begin{array}{r}9 \\
58 \\
68 \\
204 \\
263\end{array}$ & $\begin{array}{r}22 \\
232 \\
180 \\
444 \\
409\end{array}$ & $\begin{array}{r}31 \\
290 \\
248 \\
648 \\
672\end{array}$ \\
\hline
\end{tabular}

Pro : progestérone ; T : testostérone ; A : androstènedione ; DHA : déhydroépiandrostérone.

\section{Discussion.}

La première conclusion qui se dégage de la présente étude est que l'ovaire embryonnaire de Poulet, tout au moins au stade de 17 jours, forme l'œstradiol préférentiellement à l'œstrone à partir de précurseurs exogènes. Elle est corroborée par les résultats du dosage radioimmunologique dans le plasma, où, à tous les stades étudiés, entre 10 et 18 jours d'incubation, l'œstradiol prédominait (Woods et Brazzill, 1981 ; Woods et al., 1982). A cet égard, les résultats déjà signalés de Guichard et al. (1979b) trouvant après culture sur des milieux additionnés d'andostènedione ou de déhydroépiandrostérone des quantités d'œestrone plus importantes que celles d'œstradiol se trouvent donc en défaut. Mais cela ne veut pas dire que la prédominance de l'œstradiol soit la règle générale : le follicule ovarien humain produit plus d'œstrone que d'œstradiol (Smith et Ryan, 1961). Mais c'est l'inverse pour l'ovaire du Rat adulte (Chatterton et al., 1969) ou impubère (Quattropani et Weisz, 1973) ou pour l'ovaire du fœtus humain (George et Wilson, 1978).

Un petit nombre de travaux seulement semblent avoir comparé l'aptitude de divers androgènes à servir de précurseurs aux œstrogènes. D'après Gual et al. (1962), le placenta humain utiliserait la testostérone ou l'androstènedione avec une égale facilité, I'aromatisation de la déhydroépiandrostérone ayant un moins bon rendement. Par contre, comme on vient de le voir, l'ovaire embryonnaire de Poulet utilise préférentiellement la déhydroépiandrostérone, l'efficacité de la testostérone et de l'androstènedione étant moitié moindre. Cela voudrait dire que l'aromatisation de la déhydroépiandrostérone emprunte une voie métabolique ne passant ni par la testostérone ni par l'androstènedione. H serait intéressant de savoir s'il s'agit d'une particularité des Oiseaux ou des ovaires embryonnaires, dont d'autres stades devront être étudiés. II importerait aussi de rechercher les produits intermédiaires entre la déhydroépiandrostérone et les œstrogènes, la 19-OH-déhydroépiandrostérone en particulier.

Enfin, plusieurs travaux avaient rapporté la formation de testostérone par l'ovaire embryonnaire de Poulet à partir de précurseurs radioactifs ou non. $\mathrm{Ce}$ 


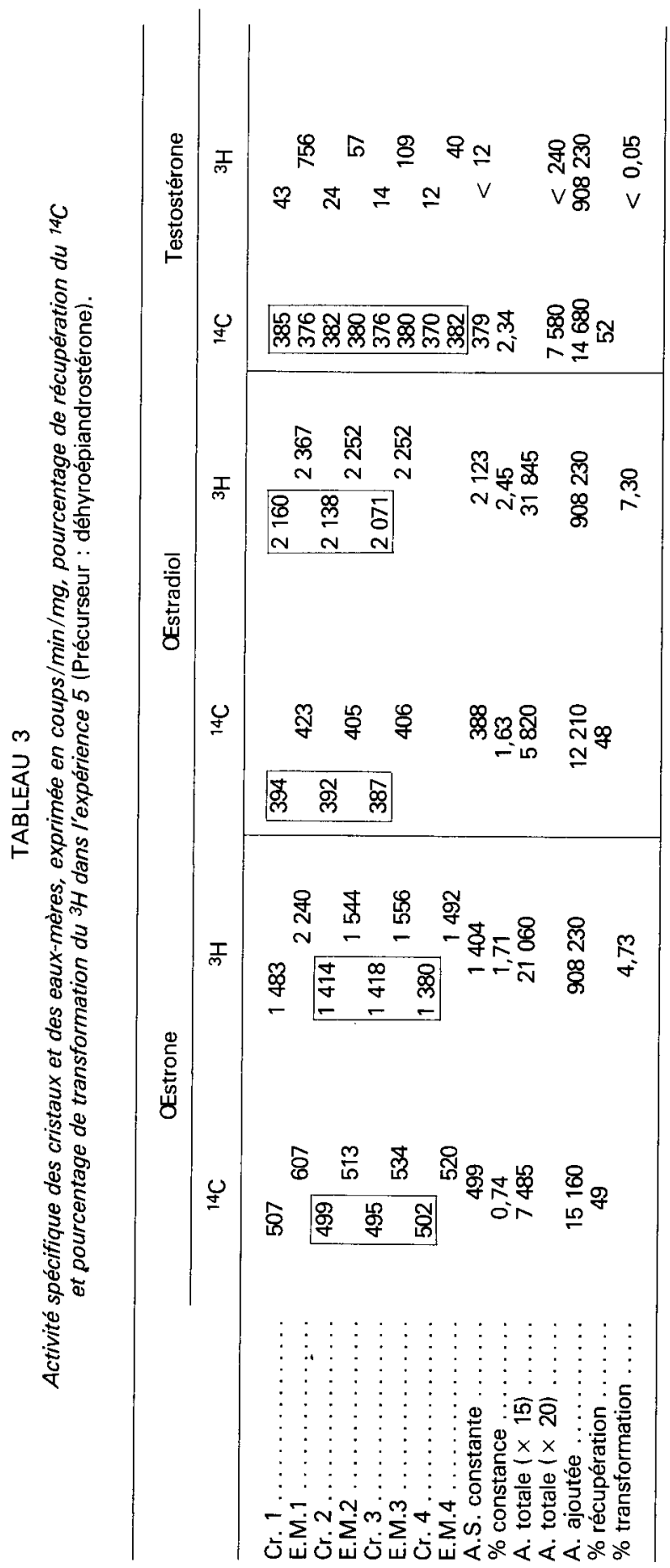


résultat n'a pas été confirmé. Etant donné les grandes quantités d'androstènedione ou de déhydroépiandrostérone présentes dans les milieux de culture, leur élimitation incomplète par chromatographie jointe à l'absence de spécificité absolue de l'immunsérum anti-testostérone pourraient expliquer les valeurs trouvées (Guichard et al., 1979b). Quant aux expériences radiochimiques, l'utilisation de méthanol aqueux pour les recristallisations n'a peut-être pas permis une purification suffisante (Guichard et al., 1973).

En résumé, le présent travail précise le métabolisme des stéroïdes par l'ovaire embryonnaire de Poulet sur les 3 points suivants : 1) La déhydroépiandrostérone est le précurseur préférentiel pour la synthèse des œestrogènes. 2) L'œstradiol prédomine sur l'œstrone. 3) II n'y a pas production de testostérone.

Reçu en mars 1983.

Accepté en juin 1983.

\section{Références}

AKRAM H., WENIGER J.-P., 1973. Effet de I'hypophysectomie sur la sécrétion d'œstrogènes par les gonades de l'embryon de Canard femelle. Gen. comp. Endocrin., 21, 543-546.

AXELROD L. R., MATTHIJSSEN C., GOLDZIEHER J. W., PULLIAM J. E., 1965. Definitive identification of microquantities of radioactive steroids by recrystallization to constant specific activity. Acta endocrin., Suppl. 99.

BROWN J. B., 1955. A chemical method for the determination of oestriol, oestrone and oestradiol in human urine. Biochem. J., 60, 185-193.

CHATTERTON R. T., CHATTERTON A. J., GREEP R. O., 1969. In vitro biosynthesis of oestrone and oestradiol-17 $\beta$ by cycling rat ovaries. Effect oi luteinizing hormone. Endocrinology, 84, 252-260.

GEORGE F. W., WILSON J. D., 1978. Conversion of androgen to oestrogen by the human fetal ovary. J. clin. Endocrin. Metab., 47, 550-555.

GUAL C., MORATO T., HAYANO M., GUT M., DORFMAN R. I., 1962. Biosynthesis of oestrogens. Endocrinology, 71, 920-925.

GUICHARD A., CEDARD L., HAFFEN K., 1973. Aspect comparatif de la synthèse de stéroïdes sexuels par les gonades embryonnaires de Poulet à différents stades du développement (étude en culture organotypique à partir de précurseurs radioactifs). Gen. comp. Endocrin., 20, 16-28.

GUICHARD A., HAFFEN K., CEDARD L., MIGNOT Th.-M., SCHEIB D., 1979a. Effects of hCG and of season on in vitro steroidogenesis by 18-day chick embryo gonads. Ann. Biol. anim. Bioch. Biophys., 19, 1317-1325.

GUICHARD A., CEDARD L., MIGNOT Th.-M., SCHEIB D., HAFFEN K., 1979b. Radioimmunoassay of steroids produced by chick embryo gonads cultured in the presence of some exogenous steroid precursors. Gen. comp. Endocrin., 39, 9-19.

HAFFEN K., CEDARD L., 1967. Métabolisme de la déhydroépiandrostérone et de la testostérone radioactives par les gonades mâles intersexuées de l'embryon de Poulet cultivées in vitro. C. R. Acad. Sci. Paris, 264, Sér. D, 1923-1926.

HAFFEN K., CEDARD L., 1968. Etude, en culture organotypique in vitro, du métabolisme de la déhydroépiandrostérone et de la testostérone radioactives par les gonades normales et intersexuées de l'embryon de Poulet. Gen. comp. Endocrin., 11, 220-234.

QUATTROPANI S. L., WEISZ J., 1973. Conversion of progesterone to oestrone and oestradiol in vitro by the ovary of the infantile rat in relation to the development of its interstitial tissue. Endocrinology, 53, 1269-1276. 
SMITH O. W., RYAN K. J., 1961. Biogenesis of oestrogens by the human ovary : formation of neutral steroid intermediates from progesterone-4- ${ }^{14} \mathrm{C}$, androstenedione-4- ${ }^{14} \mathrm{C}$ and cholesterol-4-14C. Endocrinology, 69, 970-983.

WENIGER J. -P., 1969. Recherches sur la nature chimique des hormones sexuelles embryonnaires de Poulet. Ann. Embr. Morph., 2, 433-444.

WOLFF E., HAFFEN K., 1952. Sur le développement et la différenciation sexuelle des gonades embryonnaires d'Oiseau en culture in vitro. J. exp. Zool., 119, 381-399.

WOODS J. E., BRAZZILL D. M., 1981. Plasma 17ק-oestradiol levels in the chick embryo. Gen. comp. Endocrin., 44, 37-43.

WOODS J. E., CONGORAN D. D., THOMMES R. C., 1982. Plasma oestrone levels in the chick embryo. Poultry Sci., 61, 1729-1733. 